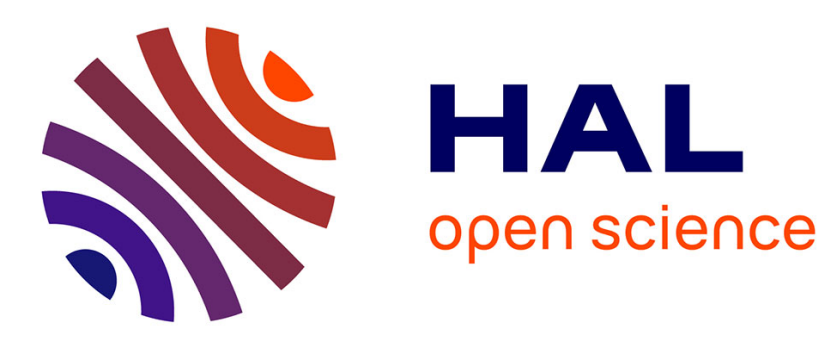

\title{
RFID Anticollision in Dense Mobile Environments
} Abdoul Aziz Mbacké, Nathalie Mitton, Hervé Rivano

\section{To cite this version:}

Abdoul Aziz Mbacké, Nathalie Mitton, Hervé Rivano. RFID Anticollision in Dense Mobile Environments. WCNC 2017 - IEEE Wireless Communications and Networking Conference, Mar 2017, San Francisco, United States. hal-01418170

\section{HAL Id: hal-01418170 \\ https://hal.inria.fr/hal-01418170}

Submitted on 8 Feb 2017

HAL is a multi-disciplinary open access archive for the deposit and dissemination of scientific research documents, whether they are published or not. The documents may come from teaching and research institutions in France or abroad, or from public or private research centers.
L'archive ouverte pluridisciplinaire HAL, est destinée au dépôt et à la diffusion de documents scientifiques de niveau recherche, publiés ou non, émanant des établissements d'enseignement et de recherche français ou étrangers, des laboratoires publics ou privés. 


\section{RFID anticollision in dense mobile environments}

\author{
Abdoul Aziz MBACKE \\ Inria, France \\ aziz.mbacke@inria.fr
}

\author{
Nathalie MITTON \\ Inria, France \\ nathalie.mitton@inria.fr
}

\author{
Herve RIVANO \\ Univ Lyon, Inria, INSA Lyon, CITI, France \\ herve.rivano@inria.fr
}

\begin{abstract}
The popularization of RFID systems has conducted to large deployments of RFID solutions in various areas under different criteria. However, such deployments, specially in dense environments, can be subject to RFID collisions which in turn affect the quality of readings. In this paper we propose two distributed and efficient solutions for dense mobile deployments of RFID systems. mDEFAR is an adaptation of a previous work highly performing in terms of collisions reduction, efficiency and fairness in dense static deployments. CORA is more of a locally mutual solution where each reader relies on its neighborhood to enable itself or not. Using a beaconing mechanism, each reader is able to identify potential (non-)colliding neighbors in a running frame and as such chooses to read or not. Performance evaluation shows high performance in terms of coverage delay for both proposals quickly achieving $100 \%$ coverage depending on the considered use case while always maintaining consistent efficiency levels above $70 \%$. Compared to GDRA, our solutions proved to be better suited for highly dense and mobile environments, offering both higher throughput and efficiency. The results reveal that depending on the application considered, choosing either mDEFAR or CORA helps improve efficiency and coverage delay.
\end{abstract}

Index Terms-RFID, reader anticollision problem, MAC layer, resource allocation, distributed systems, mobile systems

\section{INTRODUCTION}

The identification of goods has, for the past decades, been done thanks to the use of bar codes. In such systems, laser reader would have to be in direct line of sight with a given bar code to be identified. Having large warehouses where several products can be stored has made such a solution quite obsolete and even not appropriate. As such, RFID systems come to the rescue allowing for contactless and without direct line of sight identification of goods, using a technique known as back-scattering where the reader emits a radio signal towards a tag which in turn is activated/enabled and answers sending its ID. The use of radio signals and the size of tags, made it possible to have them embedded in different goods and still be identified even when disposed in lots. Such possibilities allowed new cases to come up where readers could be deployed and mobile in the system for a quicker coverage of all tags. In addition, mobile tags deployed with their successive positions being tracked by deployed readers. For instance, the case of factories could be explored. Using RFID technology in factories could allow substantial improvements regarding productivity, traceability, security. Using RFID tags deployed on each product could permit to follow all the movements of the products inside the covered area, being able to register incoming and outgoing products and somehow improve the quality of the delivery system for all goods. Having a solution that would allow the instantaneous identification of the entire content in a minimum delay would improve the traceability and reduce the burden of identifying them one by one enhancing the agility, security and productivity of the system. However, in order to establish such solutions, a very dense deployment of readers and tags need to be made. This alone creates a very collision vulnerable environment [1], but in order to further improve the system and to follow the needs of present setups, mobile readers and/or tags also need to be deployed. The addition of these latter deteriorates even more the system and thus delay.

We chose to study the design of anticollision algorithms for RFID readers taking dense deployments and mobility into account. Our proposal aims at building efficient RFID systems to reduce collisions and coverage delay while improving the throughput (in terms of tag identification).

The literature review shows different proposals [2]-[7] all highly performing in their considered environments but not acknowledging high density and/or mobility deployments. The few ones considering these characteristics are centralized approaches depending on the use of a central server for scheduling the readers [8]. This further increases the cost of the system and with the mobility taken into account, adds another needed layer of communication.

In this paper we introduce two new solutions for RFID anticollision. Both approaches are :

- distributed and local: loose TDMA approach based on readers internal clocks handling clock drifts and relying on the information in their vicinity ;

- efficient: in terms of throughput in spite of collisions ;

- mobile-ready: algorithms are reliable to mobile scenarios thanks to their local and memory-less approach.

The first proposal, mobile-Distributed Efficient and Fair Anticollision for RFID (mDEFAR) is an adaptation of a previous work to better comply with dense mobile environments by reducing the interference range considered by rendering it monochannel. The second proposal, Coverage Oriented RFID Anticollision (CORA) is a simple approach where readers accord each other locally. Some collisions are accepted in order to improve the throughput and coverage delay of the system. The concession made in terms of collisions makes CORA more suitable for less energy-constrained applications aiming at a quick coverage of deployed tags.

The rest of this paper is organized as follows, Section II reviews the problem statement and highlights our motiva- 
tion. Section III explores some state-of-the-art anticollision protocols for RFID and features their drawbacks regarding the tackled issues. Section IV details mDEFAR and CORA algorithms. Their performances relative to different metrics as well as the use cases are considered are evaluated and confronted in Section V. Finally, Section VI concludes by discussing future research directions.

\section{Problem Statement}

\section{A. Dense environments}

In order to increase the coverage area or improve the total reading delay in RFID systems, one solution was to increase the number of deployed readers. This reader densification within a given area was unfortunately not done without compromise. Indeed, the crowding of RFID readers results in the growth of the number of collisions. These latter ones can happen at different levels. For instance, when a reader tries to access multiple tags at the same time, all the interrogated tags answer at the same time resulting in a collision and the tags not being read. This type of collisions is referred to as a tag collision in the literature. They have been thoroughly studied and solved thanks to ALOHA [9], tree [10], and frame-andtree [11] based protocols.

Another instance of collisions is when multiple readers try to read a given tag at the same time. The tag, unable to dissociate signals received from multiple sources, will just consider the multiple queries as radio noise and fails to answer to any of the readers. In Fig. 2, only tags $\mathrm{T} 1$ and $\mathrm{T} 3$ will be identified by readers R1 and R2 while T2 is in the colliding area and fails to be read. In order to avoid such an issue, R1 and R2 should either operate at different times or with a distance of at least $d=2 \times d_{C R T}$ with $d_{C R T}$ being the reading range. Another solution would be to have readers operate at different frequencies [12]. However in a very dense network, the distribution of available frequencies can be quite laborious and their number not sufficient. Thus having an efficient dynamic RFID reader anticollision algorithm becomes crucial to improve tag identification. Still in the aim to increase the coverage area to fulfill the needs of new applications, readers can be mounted on ambulatory devices for mobility. Mobile readers allow a better tracking of products inside a warehouse. Deploying a mix of static and mobile readers to follow the movement of tags within a large coverage area can also be considered. However, as for the drawbacks brought by dense environments, the mobility of readers also induces new collision issues.

\section{B. Dedicated control channel}

For the sake of contention resolution, a dedicated control channel between readers is needed. Indeed, in order for readers to exchange information regarding their reading behaviors a communication channel must be set between them. The hypothesis of a dedicated control channel between readers can be seen throughout the literature [3]-[5]. Some authors also considered this communication medium to be set between the readers and a central server [8] others even contemplated the idea of a link between readers and a central server as well as a link between readers themselves [2], [13].

In both our proposals, the hypothesis is made to have a single dedicated wireless communication channel between readers to allow exchange of beacons for contention resolution. We chose to set the range of the communication channel to $d_{C O M}=d_{C R T}$. This value grants readers a knowledge of their vicinity potential colliding neighbors.

\section{RELATED WORK}

Many proposals of the literature have addressed reader collisions. They can mainly be classified in two categories: TDMA and CSMA-based. While the former relies on a prescheduled access of the readers to tags, the latter commits to listening the medium before attempting to read tags.

In TDMA-based DCS (Distributed Color Selection) [14], the readers randomly chose a slot which they use to access tags. In the event of a collision (supposing collisions can be detected), they randomly chose new slots within the maximum number of available slots. In a dense environment, this solution quickly proves to be unfitted since readers may get stuck in a new slot reservation procedure loop since the slots number might be insufficient. Willing to overcome this issue, authors then proposed VDCS (Variable DCS) [3] where the number of slots is not fix for the whole system but variable among readers. Collisions are then computed and matched to given upper and lower bounds. In case one of the bounds is reached, readers accordingly increase or decrease their maximum number of slots, chose a new one in the recently defined maximum slots number and broadcast it to their neighbors. This solution proved to resolve partially density issue but still fails to comply the constraints of mobility.

Another considered TDMA-based proposal is GDRA (Getric Distribution Reader Anticollision protocol) [2] where authors assume the presence of a central server (CS) able to communicate with all the readers at the same time. The CS broadcasts an Arrangement Command (AC) to all readers letting them know the number of slots available. Readers then randomly chose a slot and a channel according to a given geometric distribution [15]. The readers are expected to dispose of bistatic antennas allowing them to send a beacon announcing their chosen slot and channel to neighbors while receiving theirs. In case of readers sharing the same slot, they get disabled for the current round otherwise, if a reader finds no other peer with the same slot and channel as its own, it sends an Overriding Frame (OF). The OF disables all neighboring readers with no regards to chosen slot or channel for the current frame. Disabling neighbors as the OF does, induces throughput drops and uncovered tags.

Regarding CSMA proposals, we can consider Pulse. In [4], readers willing to access tags listen to the medium for a given period of time and if found idle, continuously broadcast a signal on a dedicated control channel to let the neighboring devices know that the medium is occupied. This proposal however does not fit much dense environments since several readers may get disabled for an undefined period of time to 
the benefit of a single one, thus reducing the throughput and fairness of the whole system. Also it is unqualified for mobile environments since enabled moving readers may collide.

In light of this brief review, it becomes clear that density and mobility should be taken into account in the design of an efficiently performing solution.

\section{PROPOSALS}

In regards of the considerations exposed in Section III, we designed two proposals fitting a dense and mobile RFID system. Each proposal performs differently depending on the application constraints.

\section{A. mobile-Distributed Efficient and Fair Anticollision for RFID (mDEFAR)}

mDEFAR relies on our previous work DEFAR [16] algorithm. In order to well identify the contribution of mDEFAR compared to DEFAR, we give below a brief overview.

1) DEFAR: DEFAR is a multi-channel algorithm, unlike mono-channel CORA and mDEFAR. In DEFAR, readers randomly chose a communication token defined by a reading channel and slot. After observing a backoff, readers broadcast a beacon with their tokens on a dedicated control channel (different from the reading channel). This dedicated control channel operates on a separate antenna and frequency band, as such, it cannot interfere with reading operations. According to the received tokens, each reader then decides to read or not and switches its priority for medium access accordingly for the next round. Three (3) different priorities are identified: NEUTRAL, as the priority of all readers at start, LAZY for readers that successfully accessed to the medium and PUMPED UP for readers that failed to access the medium and raise their priority for the following round. Due to the multichannel [12] feature, the communication range is set to $d_{C O M}=2 \times d_{A C}=2 \times 3,3 \times d_{C R T}$ [6] with $d_{C O M}$ the communication range between readers, $d_{A C}$ the adjacent channel interference range and $d_{C R T}$ the reader-tag interrogation range. While multiple channels allow less collisions since it is possible to have up to $N=$ max_slots $\times$ max_channels tokens, it also disables more readers since the communication range has to account for the adjacent channel interference.

2) mobile-Distributed Efficient and Fair Anticollision for RFID (mDEFAR): mDEFAR is a distributed TDMA-based RFID anticollision protocol. The larger the communication range, the more disabled readers. As such, we chose to set $d_{C O M}=d_{C R T}$ by making the proposal monochannel in order to enable more readers at the same time for dense environments. This approach allows a better throughput and coverage delay albeit the single channel parameter induces more collisions since readers shift $N=$ max_slots $\times$ max_channels available tokens to just $N^{\prime}=$ max_slots tokens.

Each slot has a duration of $T_{\text {slot }}$. This duration is split into two parts, $T_{\text {beacon }}$ [12] used by readers to send beacons at the start of their slot and $T_{C R T}$ [2], [6] used to access and read tags. A frame can then be defined as : $T_{\text {frame }}=$ max_slots $*$ $T_{\text {slot }}$ with $: T_{\text {slot }}=T_{\text {beacon }}+T_{C R T}$.
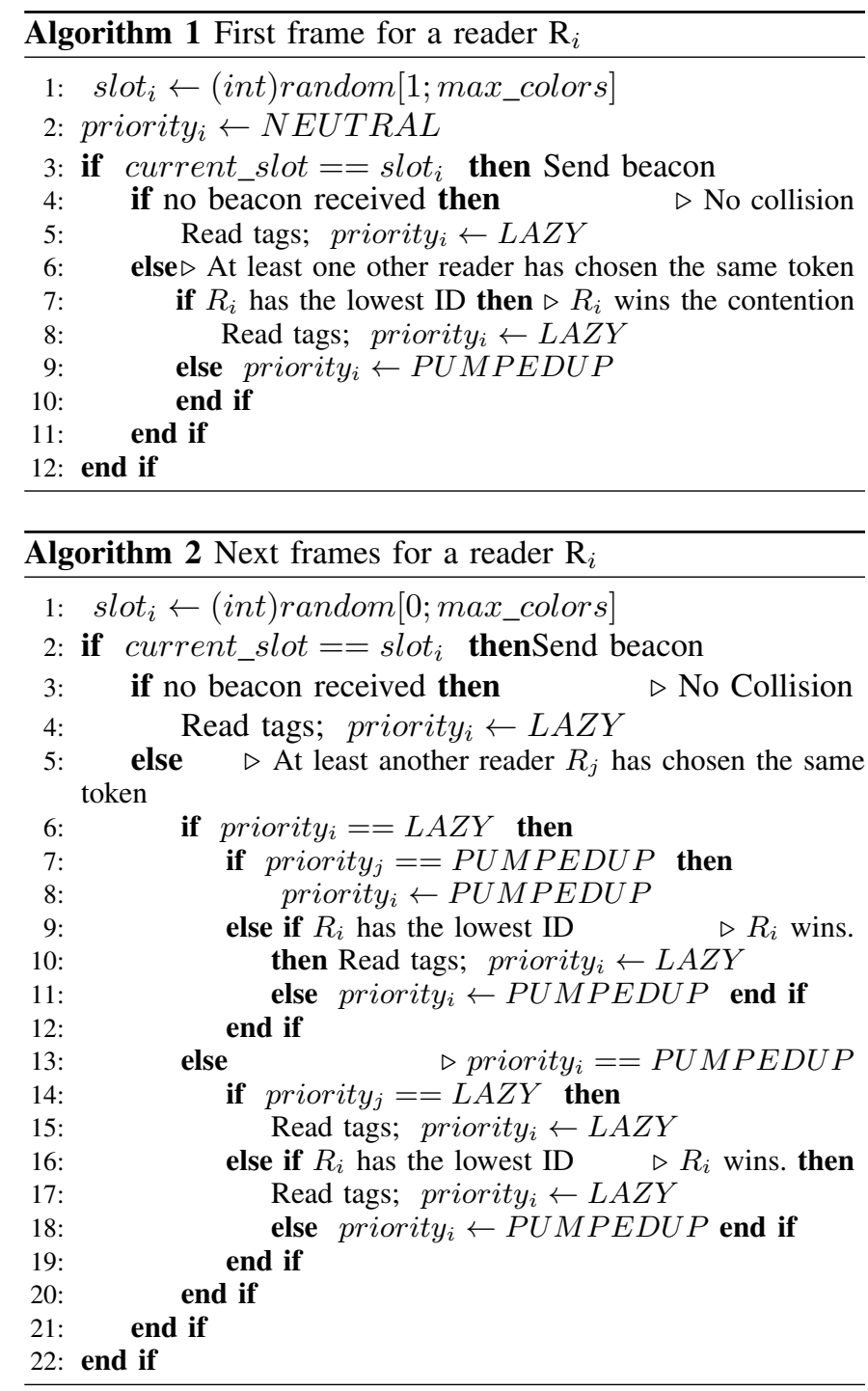

To better understand the concept of mDEFAR, let's consider the graph depicted on Fig. 1a where the readers are the vertices and the contention between them are the edges. "N" states for NEUTRAL priority and the values in brackets represent the chosen_slot. At bootstrap, readers R2, R4, R3 and R8 will collide as well as R6 and R7 and resolve their contention (Algo. 1-16). All other potential collisions are resolved by the different slots selection (Algo. 1-14). R2, R3 and R6 will win the contention since they hold the smallest IDs and will read tags (Algo. 1-17) while R4, R7 and R8 will switch to a PUMPED UP priority "P" (Algo. 1-19) and all other readers being $L A Z Y$ "L" for the next frame (Algo. 1-15-8). In the second frame (Fig. 1b), we see that R2 and R4 have chosen the same slot, as such R2 and R4 will collide (Algo. 2-15). Since R4 did not access the medium in the previous frame, it has a PUMPED UP priority and will win the contention round (Algo. 2-113-15). While R1 and R8 collide as well on slot 1, R1 loses the contention due to its lower priority (Algo. 2,168). Regarding R5, R6 and R7 choosing the same slot, on one side R5 and R6 being on the same LAZY level (Algo. 2-19) 
will compare their iD and R5 accesses tags (Algo. 2-1910). R6 being $L A Z Y$ facing $\mathrm{R} 7$ in PUMPED UP loses the round. Yet, by running mDEFAR, all readers will eventually access tags being ensured to be the only one during its slot, guarantying no collision and all tags successfully read.

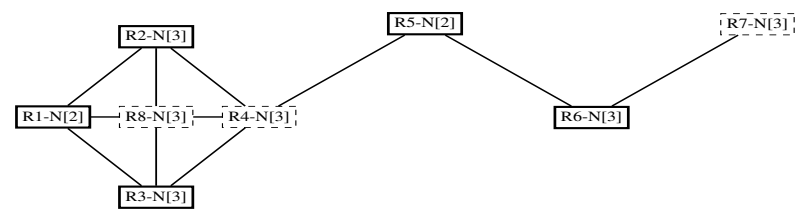

(a) $1^{\text {st }}$ frame

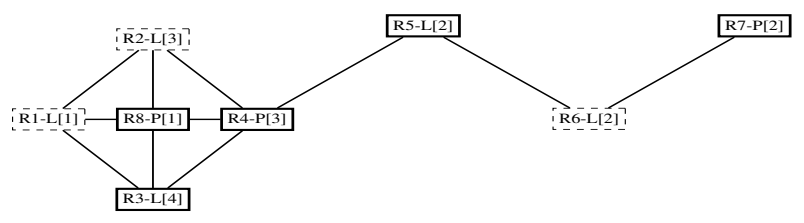

(b) $2^{\text {nd }}$ frame

Fig. 1: mDEFAR topology example

\section{B. Coverage Oriented RFID Anticollision (CORA)}

CORA is a monochannel TDMA-based proposal. For a while, RFID collisions have been considered as impactful as WSN collisions [2]-[6]. As such, it was considered that colliding readers would not access any tag and collisions were just avoided at any cost, forgetting that tags that were not in the collision area would actually be successfully read. In Fig. 2a, R1 and R2 can resp. read tags $\mathrm{T} 1$ and $\mathrm{T} 2$ while they share a collision area over $\mathrm{T} 2$. In the event of R1 and R2 accessing the medium at the same time, both tags $\mathrm{T} 1$ and $\mathrm{T} 2$ will successfully be read while $\mathrm{T} 2$ being in the collision area will not, T2 will still be read afterwards by R3 (see Fig. 2b). Thus, instead of having the three readers at three different times we can have a total coverage within just 2 timeslots improving the coverage delay.

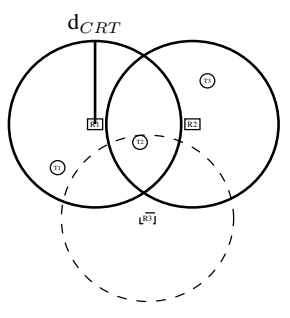

(a) Collision over tag

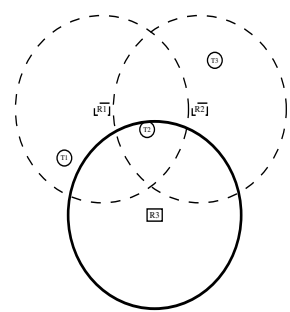

(b) $3^{\text {rd }}$ reader access
Fig. 2: Collision evaluation

The process of CORA is willingly kept simple as a monochannel and TDMA-based algorithm. The communication with tags is organized in frames themselves subdivided in slots. Every reader randomly chooses a slot within a value max_slot. The frame is designed such as the frame as a beacon phase first during which every reader broadcasts its randomly chosen slot to its vicinity and receives other's beacons, second is a tag interrogation phase. The beaconing phase is organized with a backoff scheme to prevent beacon collisions at this level. Upon reception of all beacons in its vicinity (Algo. 3-14,5), a reader then makes a decision according to the number of contenders that chose the same slot as its own (Algo. 3-16-7) and the different ones (Algo. 3-18-9). Each reader computes a number $M=$ slot $_{\text {same }}-$ slot $_{\text {different }}$.

- if $M>0$ (Algo. 3-113-14), reader considers there are too many neighbors on the same slot as its own and gets disabled. The potential size of the colliding area between all the contending readers in its vicinity involved makes it inefficient to read. In the example of Fig. 3, after beacon exchange, reader R5 will not access tags since it collides with both R4 and R6. Tags covered by R5 will be read on the following round; - if $M \leq 0$ (Algo. 3-115-16), reader accesses the medium even if it might collide with some of its neighbors considering that the uncovered tags due to collisions will be read by the neighboring readers on different slots within the same frame. As such, in Fig. 3, all readers except R5 access tags. Regarding tags laying between R2, R3 and R8, they will not be read in the current round but in the following round.

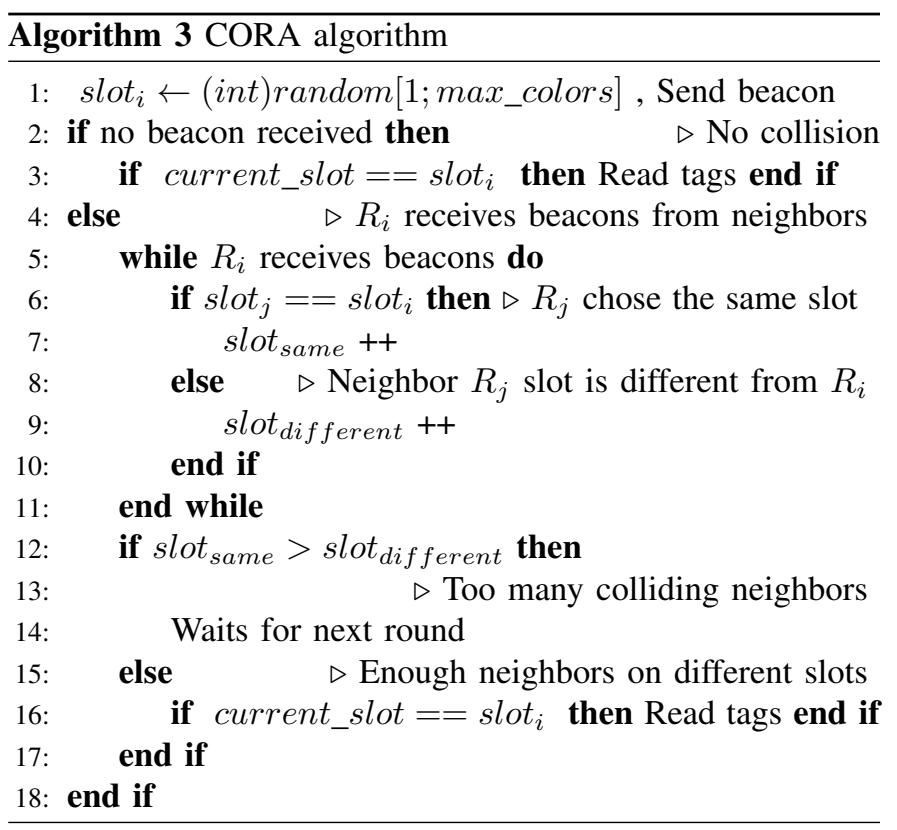

Contrarily to mDEFAR, readers here need to identify all their contending neighbors and their slots before the contention. The beaconing process is thus made prior to any reading phase. A frame has a length of: $T_{\text {frame }}=T_{\text {beacon }}+$ $\left(\max \_s l o t \times T_{C R T}\right)$.

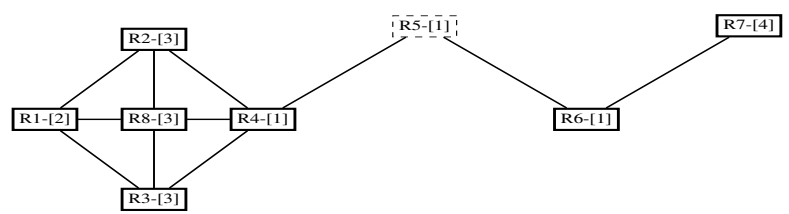

Fig. 3: CORA topology example

\section{PERformance EVAluation}

In order to evaluate our proposals, two use cases have been considered, both include density and mobility factors 
to comply with the issues of Section II. They are compared to GDRA which remains the best performing algorithm for RFID anticollision in the literature. Also, as a centralized and TDMA approach, it seemed to be the best suited to validate our distributed approaches.

\section{A. Scenarios}

1) Warehouse scenario: This scenario features a warehouse with 6000 tags deployed on shelves over $200 \times 205 \mathrm{~m}^{2}$. The shelves are considered to be aligned in rows spaced by 7 meter-wide aisles. 10 readers are deployed on each aisle, moving at a speed of $0,7 \mathrm{~m} / \mathrm{s}$ linearly within the aisles and uturn once they reach an edge. Such a scenario challenges our proposals in a dense deployment with dynamic parameters.

2) Urban scenario: This scenario features an urban representation with 800 tags used to identify moving vehicles at an average speed of $10 \mathrm{~m} / \mathrm{s}$. Static readers are placed on each building block corner. Mobile readers are mounted on bicycles riding at an average speed of $4 \mathrm{~m} / \mathrm{s}$. This model deals with the coexistence of both mobile and static readers with sporadically dense environments when mobile readers reach an intersection and are in contention with at least 4 of the corner readers.

\section{B. Performance results}

We assessed the performance of our proposals using WSNet [17], an event-driven simulator for large scale wireless networks. For each scenario, a random topology in respect to the models presented in Section V-A was generated. Each of our proposals was simulated 100 times on every model for 400s. According to the maximum transmission power defined in [12], we set the reader interrogation range $d_{C R T}=10 \mathrm{~m}$. The value of max_slot is set to 4 for a good compromise between high throughput and latency. $T_{\text {beacon }}$ and $T_{C R T}$ are resp. set to $5 \mathrm{~ms}$ and $460 \mathrm{~ms}$ according to [2], [12], [18]. The performance metrics used are defined in [19].

1) Throughput: The throughput is computed as the average number of successful query sections over the simulation length. A better throughput means readers access tags more frequently. This can be useful to qualify tracking or security applications. The figs. $4 \mathrm{a}$ and $4 \mathrm{~b}$ compare the throughput of mDEFAR, CORA and GDRA in both scenarios. In both applications, our proposals achieve better results than GDRA. Regarding the warehouse scenario, CORA has the best results since it allows collisions to happen in order to increase throughput. GDRA performs poorly because of the high density of readers deployed, the contention resolution techniques disable several readers. The same observations can be made regarding the urban scenario. The difference of throughput between CORA and MDEFAR is more noticeable since CORA offers a better contention resolution for static readers placed on corners where only one may be enabled in the case of mDEFAR against up to all 4 for CORA.

2) Collisions: Collisions are registered when multiple readers chose the same beaconing slot and when different readers in the same vicinity failed to choose different slots resulting in the case of mDEFAR, in disabled readers and in the case of
CORA, in disabled or willingly colliding readers. The number of collisions in the warehouse model between mDEFAR, CORA \& GDRA is presented in Fig. 4c while the collisions in the urban model are shown in Fig. 4d. GDRA records the highest number of collisions because of the proximity between readers. mDEFAR offers better results than CORA in the warehouse scenario thanks to the different priority levels, while CORA records collisions to increase throughput. In the urban scenario, performances of CORA and mDEFAR are similar with 20 times less collisions than GDRA.

3) Efficiency: Efficiency defines the ratio of the number of successful query sections (SQS) over the total amount of attempted query sections (AQS). Efficiency assesses how well an algorithm avoids collisions regarding the throughput it can achieve. Fig. 4e and Fig. 4f depict the computed efficiency of mDEFAR, CORA \& GDRA resp. in the warehouse model and in the urban model. While GDRA struggles to reach $1 \%$ efficiency in the warehouse scenario, both CORA and mDEFAR have efficency levels of $72 \%$ and $75 \%$ respectively. mDefar is slightly better since it registers less collisions in that scenario. In the urban scenario, GDRA performs better with the static readers and manages to reach $42 \%$, but is still far behind mDEFAR (73\%) and CORA (76\%). This implies that despite the collisions, throughput obtained by CORA makes it better fitted for this environment than mDEFAR.

4) Coverage Delay: This metric measures the speed of teh different protocols. The value is here expressed as the proportion of tags read over the number of rounds. Fig. 5 shows the coverage of our proposals in different scenarios. We notice that in the case of the warehouse, readers take more time (50 rounds) to detect all tags than in the urban scenario (20 rounds). This is due to the fact that readers only move at the speed of $0,7 \mathrm{~m} / \mathrm{s}$ thus taking long time to reach all the edges of the warehouse and designated tags. Both mDEFAR and CORA have overlapping plots, meaning similar results, and obtain full coverage after 50 rounds. GDRA, struggles to reach $10 \%$ coverage because of the low throughput and high number of collisions registered. In the urban scenario, results are slightly different, the compromise made by CORA regarding collisions allows it to reach full coverage after just 12 rounds while mDEFAR reaches $100 \%$ after 16 rounds. GDRA, although it does not reach full coverage (94\%), has significant results, this is explained by the fact that it deals better with the static readers placed in the street corners but has a hard time scheduling the moving readers. The results observed corroborate the direction chosen with CORA regarding the willingly endured collisions in order to improve delay which here is significantly lower than one's of mDEFAR.

\section{Discussions}

Note that the gap in performance is explained by the inability for GDRA to deal with highly dense or mobile environments. Indeed, our proposals were designed from scratch for these constraints. It thus allows a certain level of interference for a better coverage, in the case of CORA for example, or different priorities level to induce greater fairness 


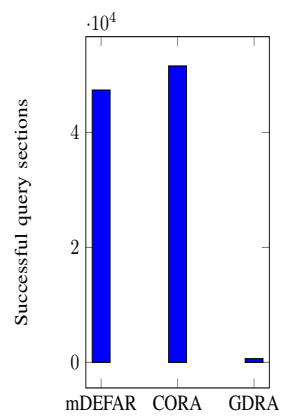

(a) Warehouse - THP

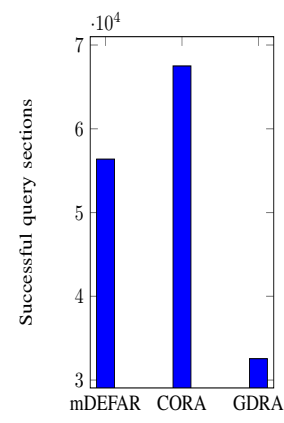

(b) Urban - THP

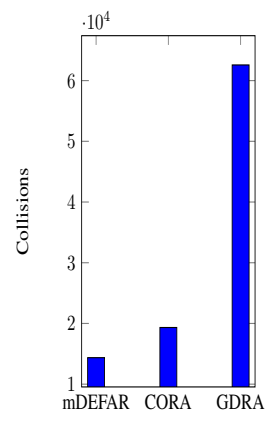

(c) Warehouse - COL

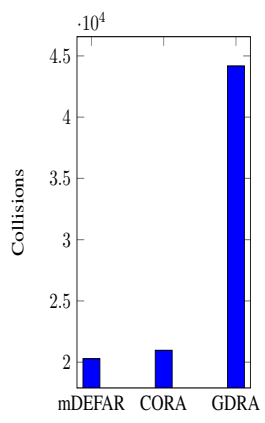

(d) Urban - COL

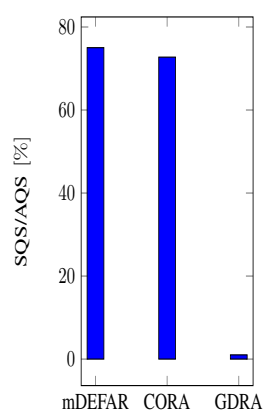

(e) Warehouse - EFF

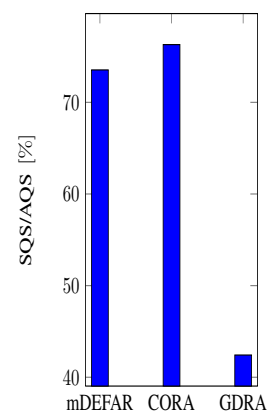

(f) Urban - EFF

Fig. 4: Results

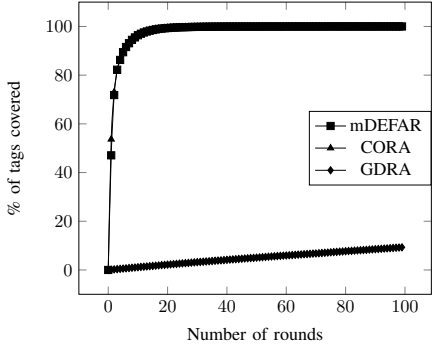

(a) Warehouse

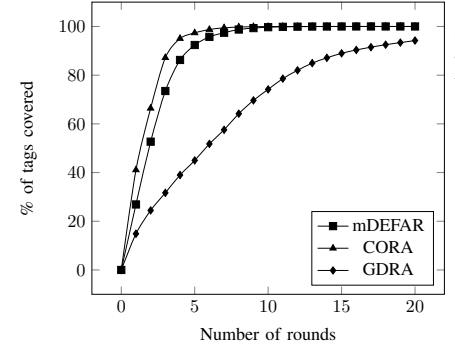

(b) Urban
Fig. 5: Coverage delay

between devices, in the case of mDEFAR. The contention resolution process introduced by GDRA is also responsible for this high level of disabled readers. In case of a collision, all involved readers skip the current round and wait for the following contention. For the tested applications, this results in a high number of collisions and low throughput. The observed results confirm the belief that an all-around solution for RFID anticollision cannot be considered. As such, for a dynamic warehouse with mobile readers and tags, mDEFAR should be chosen for its efficiency, but if the goal is to offer the fastest coverage and higher throughput, CORA is the best choice. While in the case of a hybrid deployment of both mobile and static readers, CORA turns out to be the best in terms of efficiency, so is therefore better suited for long-term stability and quick coverage, while mDEFAR should be chosen if the system is vulnerable to collisions.

\section{CONCLUSION}

We introduced two new distributed TDMA-based RFID anticollision algorithms. mDEFAR is a previous work improvement to fit mobility and high density deployments of RFID readers. CORA leverages on the particularity of RFID collisions to improve throughput and coverage delay. Both are compliant with [12] standard allowing their potential use all around different environments. Where CORA seems to be a better solution for applications requiring a quicker coverage of tags, it also shows lower efficiency than mDEFAR and thus might not be more suitable for energy-constrained systems.
Next steps will investigate the energy consumption of both approaches as well as the energy cost of collisions in order to leverage on the compromise made by CORA.

\section{REFERENCES}

[1] EPC TM radio-frequency identity protocols class-1 generation-2 UHF RFID protocol for communications at $860 \mathrm{MHz}-960 \mathrm{MHz}$ version 1.2.0, EPCglobal Standard specification Std. 1.2.0, 2007.

[2] M. V. Bueno-Delgado, R. Ferrero, F. Gandino, P. Pavon-Marino, and M. Rebaudengo, "A geometric distribution reader anti-collision protocol for RFID dense reader environments," IEEE Trans. on Automation Science and Engineering, 2013.

[3] J. Waldrop, D. W. Engels, and S. E. Sarma, "Colorwave: an anticollision algorithm for the reader collision problem," in Proc. of ICC, 2003.

[4] S. Birari and S. Iyer, "Pulse : A mac protocol for rfid networks," in Proc. of Int. workshop on RFID and Ubiquitous Sensor Networks (USN), 2005.

[5] J. Yu and W. Lee, "GENTLE: Reducing reader collision in mobile RFID networks," in Proc. of MSN, 2008.

[6] I. Amadou and N. Mitton, "HAMAC: High adaptive MAC protocol for dense RFID reader-to-reader networks," in Proc. of AdHocNets, 2015.

[7] G. P. Joshi, K. M. A. Mamun, and S. W. Kim, "A reader anti-collision MAC protocol for dense reader RFID system," in Proc. of CMC, 2009.

[8] M. Xia, Q. Yu, and Z. Li, "Relative density based anti-collision algorithm in RFID networks with dense readers," in Proc. of TENCON, 2015.

[9] G. Khandelwal, K. Lee, A. Yener, and S. Serbetli, "ASAP: a MAC protocol for dense and time-constrained RFID systems," EURASIP journal on Wireless Communications and Networking, 2007.

[10] J. Myung, W. Lee, and J. Srivastava, "Adaptive binary splitting for efficient RFID tag anti-collision,” IEEE Communications Letters, 2006.

[11] J.-B. Eom, T.-J. Lee, R. Rietman, and A. Yener, "An efficient framedslotted ALOHA algorithm with pilot frame and binary selection for anti-collision of RFID tags," IEEE Communications Letters, 2008.

[12] Electromagnetic compatibility and Radio spectrum Matters ERM Radio Frequency Identification Equipment operating in the band $865 \mathrm{MHz}$ to $868 \mathrm{MHz}$ with power levels up to $2 \mathrm{~W}$ Part 2, ETSI Std., 2011.

[13] R. Ferrero, F. Gandino, B. Montrucchio, and M. Rebaudengo, "A fair and high throughput reader-to-reader anticollision protocol in dense RFID networks," Proc. Trans. on Industrial Informatics, 2012.

[14] J. Waldrop, D. W. Engles, and S. E. Sarma, "Colorwave: An anticollision algorithm for the reader collision problem," in Proc. of ICC, 2003.

[15] K. Jamieson, H. Balakrishnan, and Y. Tay, "Sift: A MAC protocol for event-driven wireless sensor networks," in Wireless Sensor Networks. Springer, 2006.

[16] A. A. Mbacke, N. Mitton, and H. Rivano, "Distributed efficient \& fair anticollision for RFID protocol," in Proc. of WiMob, 2016.

[17] WSNet - an event-driven simulator for large scale wireless sensor networks. [Online]. Available: http://wsnet.gforge.inria.fr/

[18] I. Amadou, A. A. Mbacké, and N. Mitton, "How to improve csma-based MAC protocol for dense RFID reader-to-reader networks?" in Proc. of ADHOC-NOW, 2014.

[19] F. Gandino, R. Ferrero, B. Montrucchio, and M. Rebaudengo, "Evaluation criteria for reader-to-reader anti-collision protocols," Politecnico di Torino, Tech. Rep., 2012. 